\title{
Overproduced Brucella abortus PdhS-mCherry forms soluble aggregates in Escherichia coli, partially associating with mobile foci of IbpA-YFP
}

\author{
Charles Van der Henst ${ }^{1}$, Caroline Charlier ${ }^{2}$, Michaël Deghelt ${ }^{1}$, Johan Wouters ${ }^{2}$, Jean-Yves Matroule ${ }^{1}$, \\ Jean-Jacques Letesson ${ }^{1}$, Xavier De Bolle ${ }^{1^{*}}$
}

\begin{abstract}
Background: When heterologous recombinant proteins are produced in Escherichia coli, they often precipitate to form insoluble aggregates of unfolded polypeptides called inclusion bodies. These structures are associated with chaperones like IbpA. However, there are reported cases of "non-classical" inclusion bodies in which proteins are soluble, folded and active.

Results: We report that the Brucella abortus PdhS histidine kinase fused to the mCherry fluorescent protein forms intermediate aggregates resembling "non-classical" inclusion bodies when overproduced in E. coli, before forming "classical" inclusion bodies. The intermediate aggregates of PdhS-mCherry are characterized by the solubility of PdhS-mCherry, its ability to specifically recruit known partners fused to YFP, suggesting that PdhS is folded in these conditions, and the quick elimination (in less than $10 \mathrm{~min}$ ) of these structures when bacterial cells are placed on fresh rich medium. Moreover, soluble PdhS-mCherry foci do not systematically colocalize with IpbA-YFP, a marker of inclusion bodies. Instead, time-lapse experiments show that IbpA-YFP exhibits rapid pole-to-pole shuttling, until it partially colocalizes with PdhS-mCherry aggregates.
\end{abstract}

Conclusion: The data reported here suggest that, in E. coli, recombinant proteins like PdhS-mCherry may transit through a soluble and folded state, resembling previously reported "non-classical" inclusion bodies, before forming "classical" inclusion bodies. The dynamic localization of IbpA-YFP foci suggests that the IbpA chaperone could scan the E. coli cell to find its substrates.

\section{Background}

Escherichia coli is widely used to produce recombinant proteins of interest. One of the major concerns in the overproduction process is the formation of insoluble structures called inclusions bodies (IB) [1,2]. IB formation results from the aggregation of misfolded polypeptides that have escaped quality control by chaperones and proteases to interact through their exposed hydrophobic regions before precipitating [3]. Aggregate formation and features are influenced by various growth conditions such as temperature and $\mathrm{pH}$ [4], culture phase [5] and glucose/oxygen availability [6].

\footnotetext{
* Correspondence: xavier.debolle@fundp.ac.be

'Molecular Biology Research Unit (URBM), University of Namur (FUNDP), 61 rue de Bruxelles, 5000 Namur, Belgium

Full list of author information is available at the end of the article
}

In vivo protein aggregation is a dynamic reversible process [7]. Chaperones involved in aggregate dissociation, e.g. DnaK/DnaJ/ClpB and IbpA/IbpB, colocalize with IB in E. coli [8-11]. Recently, it has been reported that aggregate cellular localization is not random [9]. Small protein aggregates are delivered to a cell pole to form larger structures that are further dissolved by an energy dependent process [12]. All proteins in IB were initially considered as unfolded, but it has been shown that some polypeptides inside aggregates are present in an active form $[2,13,14]$. Several groups reported the formation of "non-classical" IB mainly characterized by the presence of folded and soluble recombinant proteins $[15,16]$.

Here, we report a novel example of "non-classical" IB that contain folded and soluble recombinant proteins and only transiently interact with the IpbA chaperone.

\section{C) Biomed Central}


Indeed, overproduction of Brucella abortus PdhS cytoplasmic histidine kinase [17] in E. coli revealed that PdhS-mCherry fusions were first folded and soluble in aggregates formed during the stationary phase of culture before forming insoluble structures having all the characteristics of "classical" IB. These "classical" IB recruited IpbA-YFP, as previously reported for other IB in E. coli [11], unlike the intermediate "non classical" IB. We observed that IbpA-YFP was able to form foci with very dynamic properties inside E. coli and to reach and colocalize with soluble PdhS-mCherry aggregates.

\section{Results}

PdhS-mCherry forms growth phase-dependent aggregates in $E$. coli

We used the pCVDH07 plasmid to overexpress the $p d h S$ coding sequence (CDS) fused in frame with the CDS for the fluorescent reporter mCherry (see Materials and Methods). Interestingly, the localization of this fusion in $E$. coli revealed foci at quarter (2\%), mid-cell $(10 \%)$ and polar (88\%) sites of E. coli S17-1 in the stationary phase $(\mathrm{n}=200)$ (Fig. 1B). The PdhS-mCherry was a stable fusion in E. coli, since Western blot analysis using antibodies raised against mCherry revealed a major band with the expected molecular mass for the complete fusion (data not shown). Fusing the $p d h S$ CDS to the $y f p$ or $c f p$ CDS on the same backbone plasmid or overexpressing the pdhS-mCherry fusion in DH10B, TOP10 and MG1655 E. coli strains also generated similar fluorescent foci (data not shown). When a pdhS$m$ Cherry fusion was carried on a low-copy plasmid, there was no polar focus in E. coli, contrary to its expression in B. abortus where PdhS-mCherry monopolar foci were present (data not shown). Other B. abortus proteins (the DivK response regulator, FumA and FumC fumarases) fused to the mCherry $\mathrm{N}$-terminus did not generate fluorescent foci but rather a diffuse signal (data not shown). Taken together, this data suggests that foci formation in E. coli is mainly due to PdhS itself and to the abundance of the whole PdhS-mCherry recombinant protein.

Given that bacteria growth conditions strongly influence aggregate formation, we checked whether the fluorescent foci were dependent on the growth phase, as
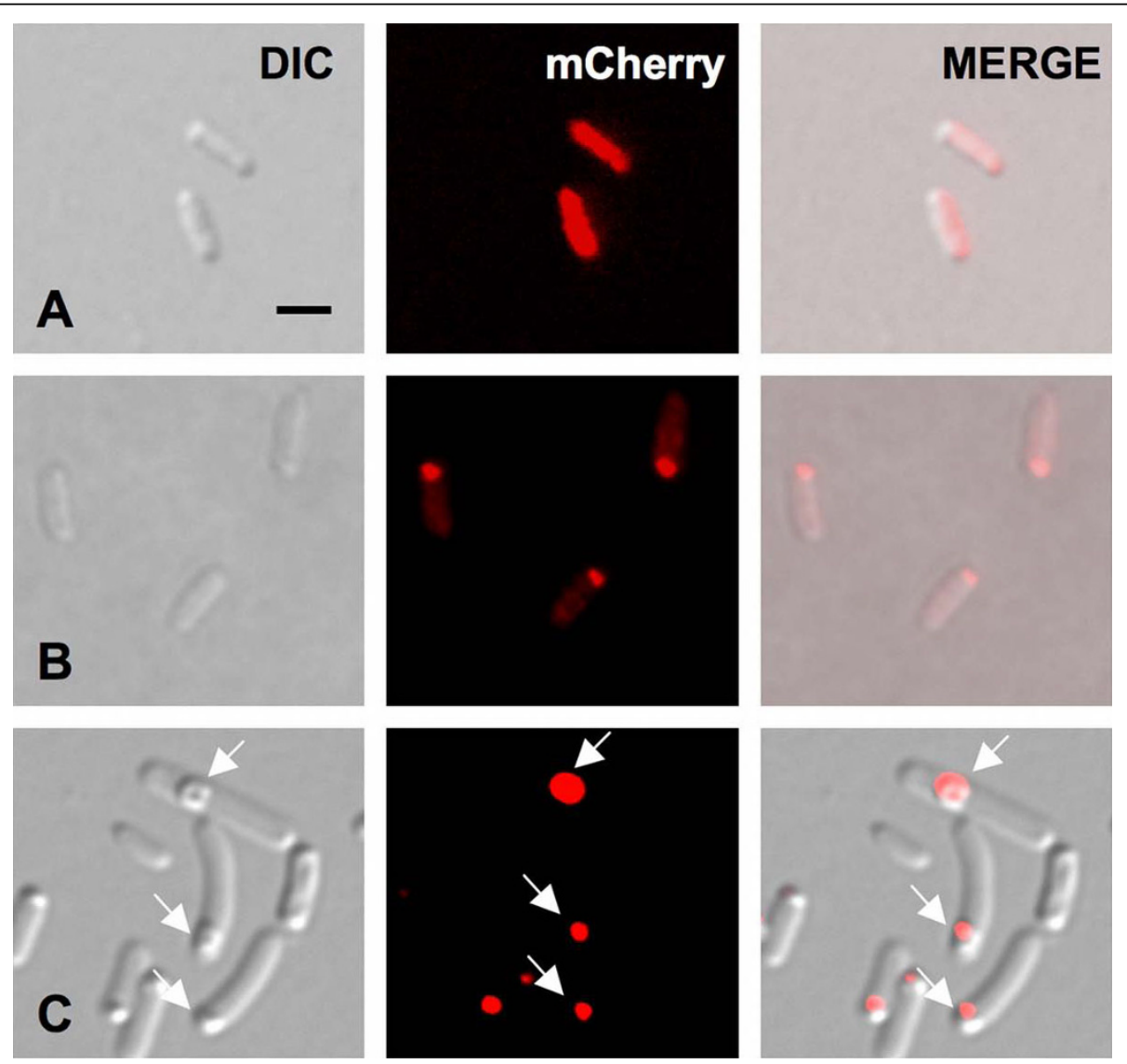

Figure 1 Fluorescent distribution of PdhS-mCherry fusion in stationary growth phase $E$. coli. A, early stationary phase; $\mathbf{B}$, middle stationary phase; $\mathbf{C}$, late stationary phase. White arrows point to refractile bodies that are only present in the bacteria from the late stationary culture phase. Scale bar: 2 Mm. DIC means differential interference contrast (Nomarski). All micrographic images were taken with the same magnification. 
previously reported for IB [5]. Using the pdhS-mCherry overexpressing strain, we observed bacteria grown until the early, mid and late stationary phase, corresponding to bacteria having just reached the maximal turbidity of the culture $\left(\mathrm{t}_{0}\right)$, the bacteria $12 \mathrm{~h}$ later $\left(\mathrm{t}_{12}\right)$, and the bacteria $36 \mathrm{~h}$ later $\left(\mathrm{t}_{36}\right)$, respectively. At $\mathrm{t}_{0}$ of the stationary culture phase, very few bacteria $(4 \%, n=100)$ showed polar fluorescent foci as many were associated with a bright diffuse cytoplasmic fluorescent signal (Fig. 1A). Twelve hours later in the same medium $\left(t_{12}\right)$, polar fluorescent foci were observed (in 98\% of the observed bacteria, $n=100$ ), together with a decrease of the diffuse cytoplasmic fluorescent signal (Fig. 1B). No detectable refractile bodies were observed in these conditions. After 24 additional hours $\left(t_{36}\right)$, larger and brighter fluorescent polar foci were formed, colocalizing with dense refractile bodies typical of "classical" IB, and accompanied by a strong decrease of the diffuse fluorescent signal (Fig. 1C).

When stationary phase bacteria (at $t_{12}$ ) showing polar fluorescent PdhS-mCherry aggregates were placed on an agarose pad made with rich medium (LB), fluorescent structures quickly disappeared (in less than 10 minutes) (Fig. 2A). Interestingly, when bacteria of the same culture were placed on an agarose pad containing phosphate buffered saline (PBS), fluorescent foci were still detectable for 3 hours and even increased in size and intensity with the appearance of dense refractile bodies (Fig. 2B). Fluorescence decrease in rich medium did not result from photobleaching, since fluorescence was still detectable after repeat exposure of bacteria on agarose pads without additional rich medium. The "classical" IB present in late stationary phase bacteria $\left(\right.$ at $\left.\mathrm{t}_{36}\right)$ were still observable when these bacteria were placed on an agarose pad supplemented with LB rich medium (Fig. 2C) or PBS (data not shown). Together, these data suggest that fluorescent foci observed during the mid stationary phase are reversible and different from those observed during the late stationary phase of culture.

\section{Colocalization assays between PdhS-mCherry fluorescent aggregates and IbpA-YFP fusions}

IbpA (for Inclusion body protein A) is a small heat shock chaperone discovered in E. coli [8]. The IbpAYFP fusion was already successfully used to label inclusion bodies in vivo, in single cells of $E$. coli [11]. As PdhS-mCherry fluorescent polar foci generated during the mid and late stationary culture phases could differ from each other, we tested their possible colocalization with the IbpA-YFP fusion.

We transformed the pCVDH07, to overexpress the $p d h S$ - $m$ Cherry fusion, in a strain expressing a chromosomal $i b p A-y f p$ fusion, previously used to monitor aggregates in vivo [11]. Using fluorescence microscopy, we observed the PdhS-mCherry aggregates and IbpAYFP localization in early, mid and late stationary phase bacteria (Fig. 3). During the early stationary phase $\left(t_{0}\right)$, the bacteria displayed a diffuse cytoplasmic PdhSmCherry signal while IbpA-YFP foci were mainly present at the cell poles (Fig. 3A). Surprisingly, in mid

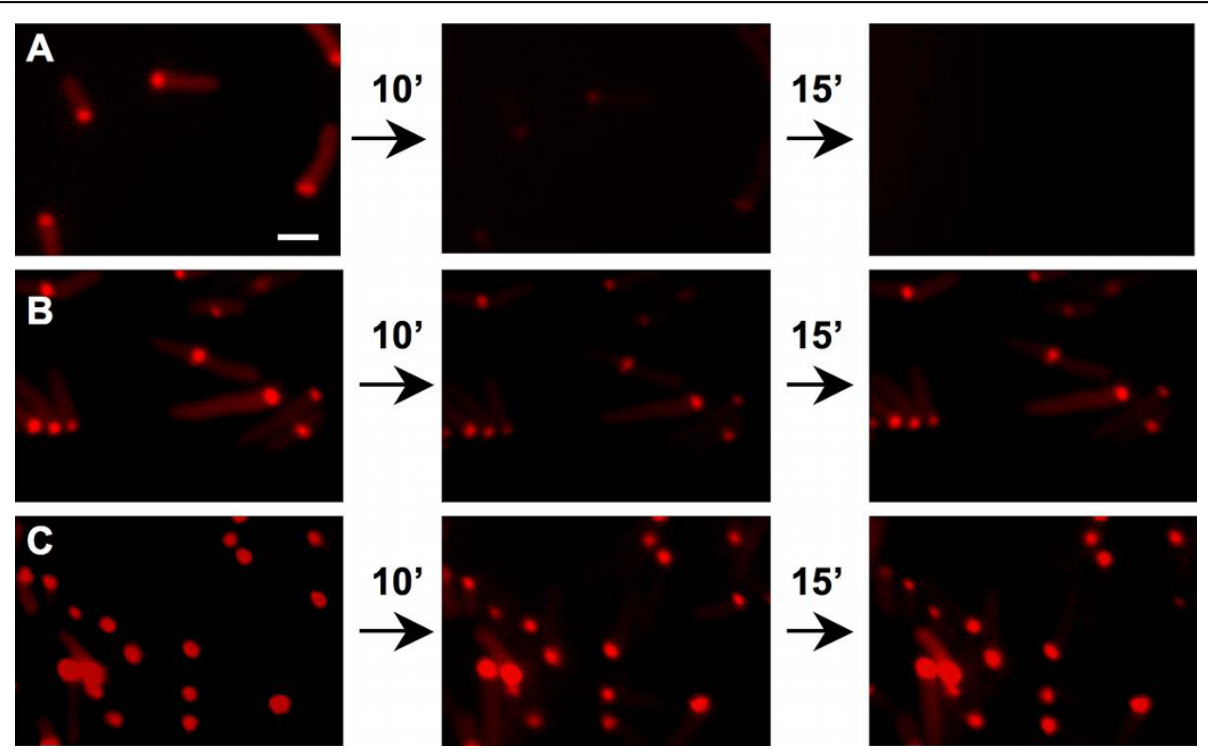

Figure 2 Stability of PdhS-mCherry aggregates in E. coli grown until the stationary culture phase. Fluorescent micrographic images taken using TxRed filter to visualize mCherry fluorescence. Pictures were taken using the same parameters, at intervals of 10 and 15 min, as indicated. A, middle stationary phase bacteria on agarose pad supplemented with LB medium; $\mathbf{B}$, middle stationary phase bacteria on agarose pad with PBS; C, late stationary phase on LB medium. Scale bar: 2 $\mu \mathrm{m}$. All micrographic images were taken with the same magnification. 


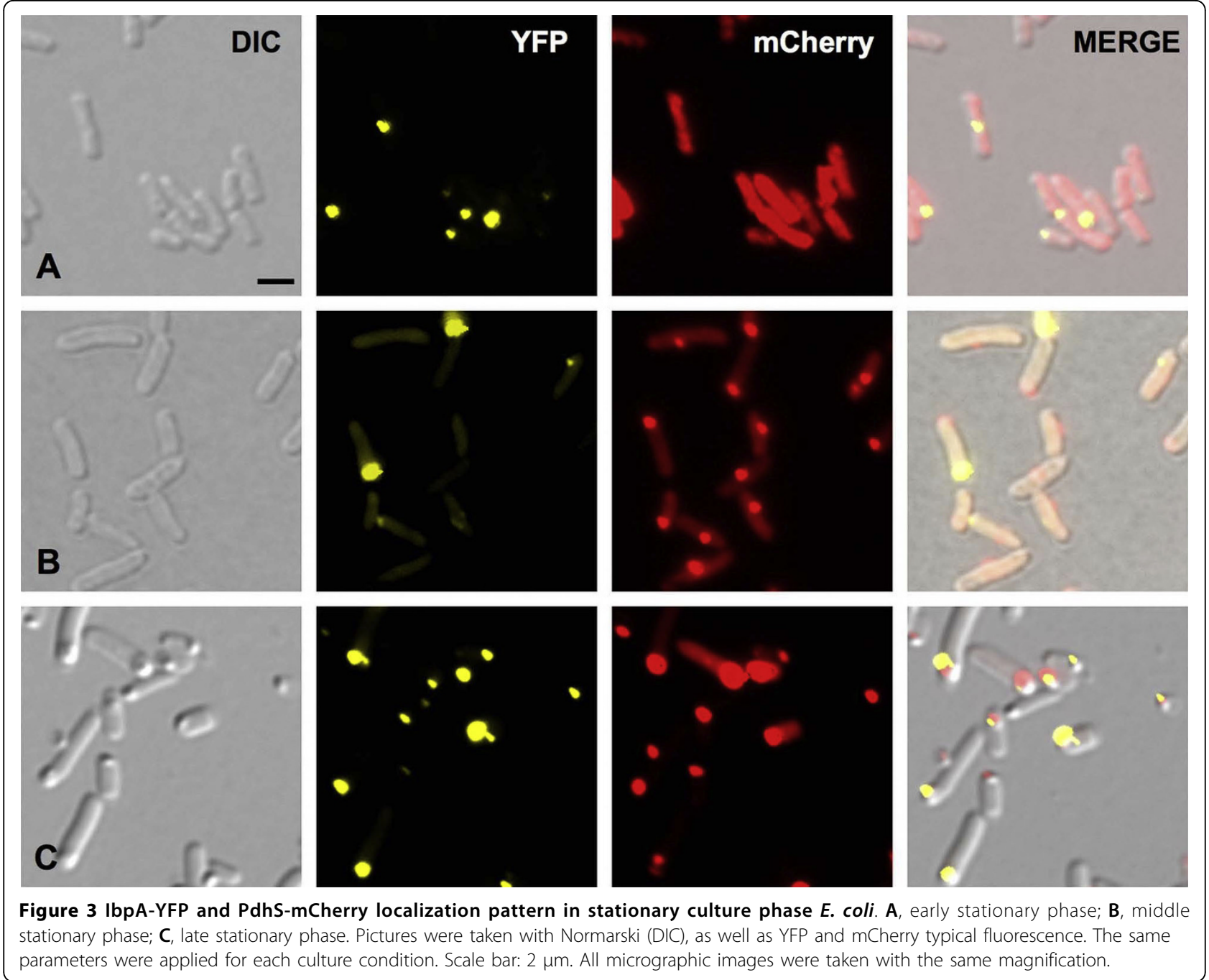

stationary phase bacteria $\left(t_{12}\right)$, colocalization of PdhSmCherry with IbpA-YFP was quite rare (Fig. 3B). Indeed, only $15 \%$ of these bacteria $(n=250)$ displayed the two corresponding fluorescent foci at the same poles, $15 \%$ at the opposite pole, $15 \%$ at an intermediate position (often near midcell) and, in $60 \%$ of these bacteria, only one fluorescent focus corresponding to PdhS-mCherry was detectable. Moreover, in the bacteria with both fluorescent signals at the same pole, we systematically observed that PdhS-mCherry and IbpAYFP did not exactly overlap (Fig. 4). At a later stage of the stationary culture phase $\left(t_{36}\right)$, polar colocalization of PdhS-mCherry and IbpA-YFP was frequent (78\%) (Fig. 3C), but the signal of both fluorescent fusions was also slightly shifted. In these late stationary phase bacteria, both foci also colocalized with dense refractile bodies seen in differential interference contrast (DIC) (Fig. 3C). At $t_{36}$, the polar IbpA-YFP foci were more frequent and were larger and brighter compared with non-polar IbpA-YFP foci. Western blot analyses showed that the IbpA-YFP fusion was not cleaved (data not shown).

Time-lapse experiments were performed to monitor the kinetics of the cytoplasmic distribution of PdhSmCherry and IbpA-YFP fusions. Mid stationary growth phase bacteria $\left(t_{12}\right)$ were plated on LB agarose pads and observed every two minutes at $37^{\circ} \mathrm{C}$ (see Materials and Methods). We observed a very dynamic localization pattern of IbpA-YFP foci in bacteria that did not contain a PdhS-mCherry aggregate (Fig. 5A). In contrast, when the PdhS-mCherry aggregate was present in $t_{12}$ bacteria, IbpA-YFP foci moved from pole to pole until they colocalized with the immobile PdhS-mCherry foci (movie S1, Fig. 5B and 5C), which in turn progressively disappeared, as previously observed (Fig. 2). In the late stationary phase cultures, the large IbpA-YFP polar clusters colocalizing with PdhS-mCherry did not move (data not shown). 


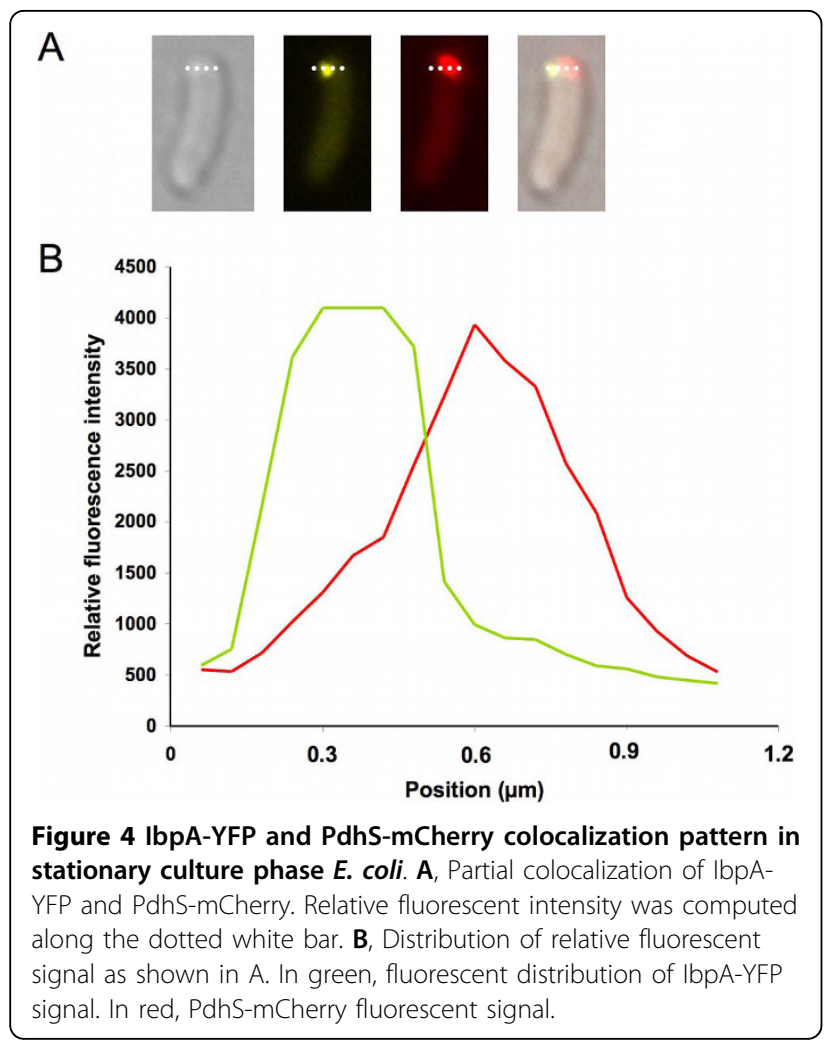

PdhS-mCherry fusions in fluorescent foci of mid stationary phase cells display properties of folded proteins

Since the PdhS-mCherry foci observed during the mid stationary phase did not colocalize with IbpA-YFP, it was tempting to speculate that PdhS-mCherry fusions were correctly folded in these aggregates. In keeping with this idea, Western blot analysis using anti-mCherry antibodies showed that PdhS-mCherry was mainly found in the soluble fraction of bacteria grown until the late stationary phase (Additional file 2, Figure S1). When soluble extracts were examined by gel permeation combined with fluorescence and Western blot analysis, soluble PdhS-mCherry proteins were identified as a single peak, with a predicted molecular weight between $669 \mathrm{kDa}$ and $20,000 \mathrm{kDa}$, the upper limit of the fractionation range (Additional file 2, Figure S2). This suggests that the fusion is able to form multimers with a defined number of monomers, further implying that PdhSmCherry is folded.

Using yeast two-hybrid assays, it was recently shown that B. abortus PdhS was able to interact with FumC through its amino-terminal domain [18], and with DivK through its carboxy-terminal domain [17]. Interestingly, FumC from Caulobacter crescentus did not interact with B. abortus PdhS [18]. When B. abortus FumC-YFP and
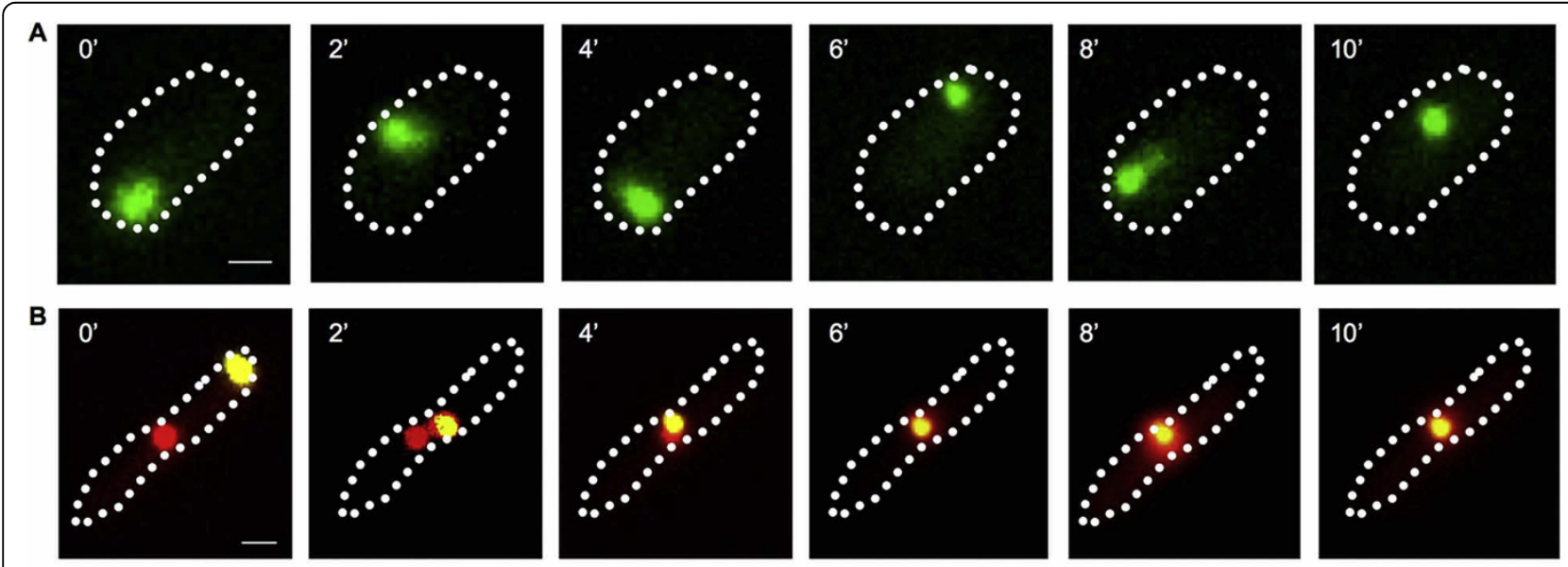

C
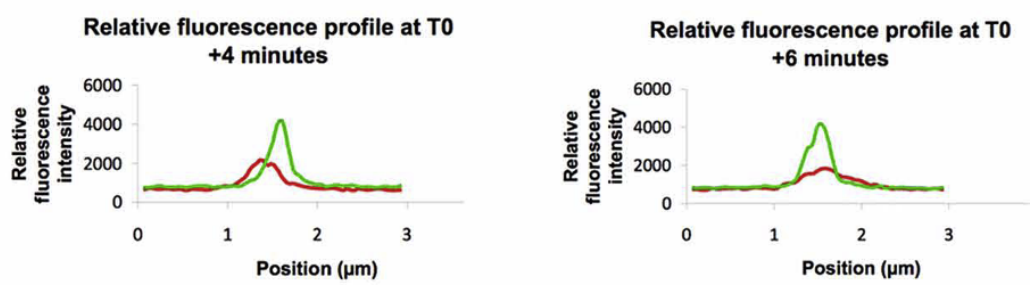

Figure 5 Dynamic localization pattern of IbPA-YFP in stationary growth phase $\boldsymbol{E}$. coli. Fluorescent micrographic images of middle stationary phase bacteria plated on rich medium taken every 2 minutes. A: IbpA-YFP; B: IbpA-YFP (yellow) and PdhS-mCherry (red). C: Fluorescence intensity of IbpA-YFP (green) and PdhS-mCherry (red) fusions at times T0, T0+4 minutes and T0+6 minutes. Scale bar: $1 \mu \mathrm{m}$ 


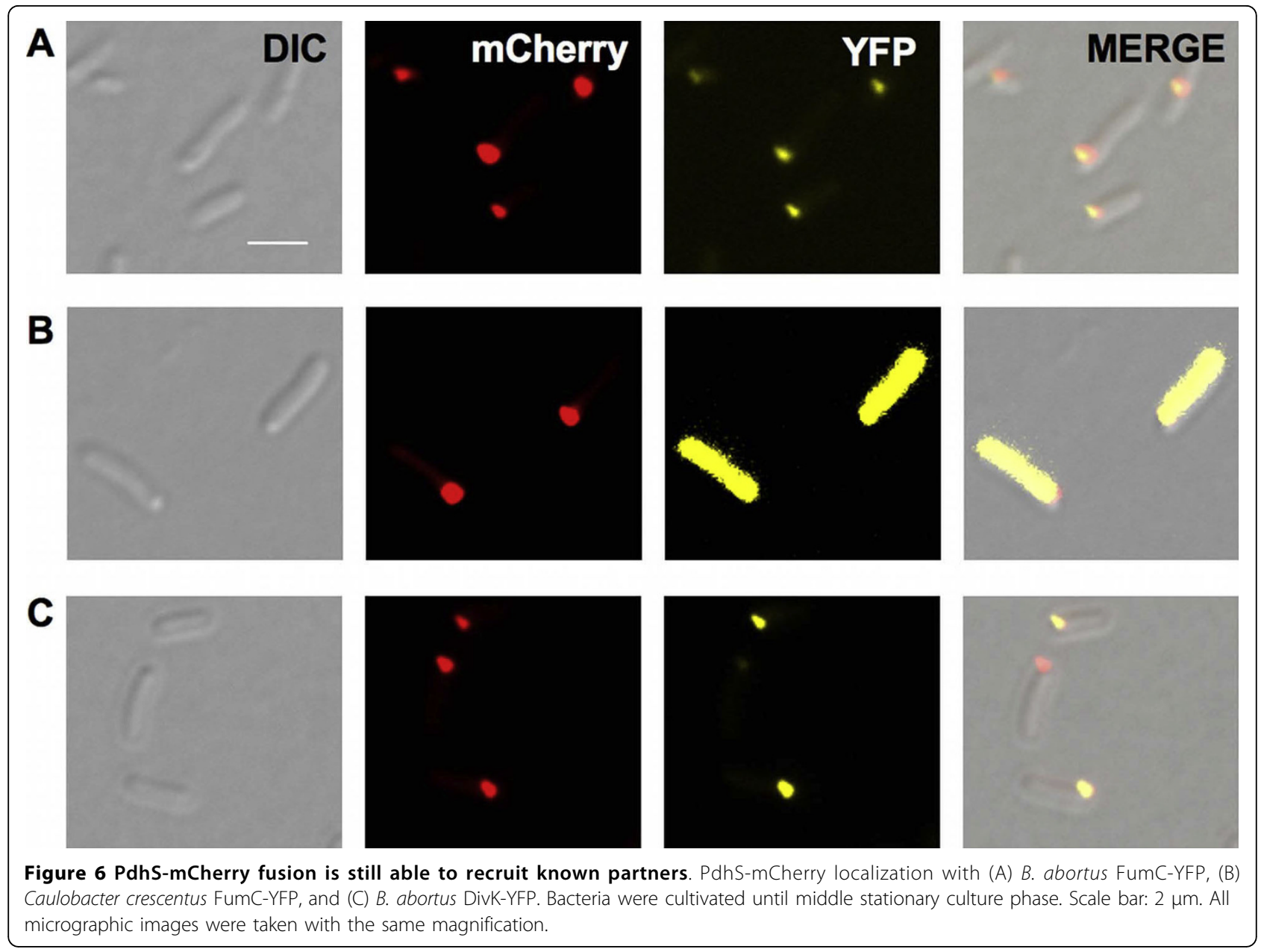

DivK-YFP fusions were produced with PdhS-mCherry, colocalization of YFP and mCherry fluorescence signals was observed in mid stationary phase E. coli cells (Fig. $6 \mathrm{~A}, \mathrm{C}$ ). Interestingly, both fluorescence signals were overlapping, further suggesting that the shift in fluorescence signals observed between PdhS-mCherry and IbpA-YFP (Fig. 4) was not an artefact. As a control, we checked that $C$. crescentus FumC did not colocalize with PdhS-mCherry (Fig. 6B). The ability of PdhS-mCherry to recruit $B$. abortus DivK-YFP and FumC-YFP but not $C$. crescentus FumC-YFP suggests that the $\mathrm{N}$-terminal and C-terminal domains of PdhS were at least partially folded.

\section{Discussion}

We report that, when overproduced in E. coli, B. abortus PdhS fused to mCherry is able to form intermediate aggregates of soluble proteins resembling previously reported "non-classical" IB [3,15], before forming "classical" IB. These intermediate aggregates are very different from "classical" IB because they are soluble, are quickly removed when bacteria are placed in rich medium (Fig.
2A), do not systematically colocalize with IbpA-YFP (Fig. 3B) and are still able to recruit known PdhS partners (Fig. 6). The observation of "intermediate" aggregates of soluble proteins does not fit with a simple model of IB formation in which unfolded proteins precipitate to form IB immediately after translation. Our observations thus suggest that some proteins could form aggregates of folded and soluble polypeptides before their precipitation into "classical" IB. The initial solubility of heterologous PdhS-mCherry could be due to a slow expression (as suggested by the slow accumulation of PdhS-mCherry, Additional file 2, Figure S1), since there is no predicted promoter identified upstream the CDS in the pCVDH07 plasmid, and the codon bias of $p d h S$ CDS is probably not optimal for sustained translation. Strong accumulation could lead to the saturation of chaperones and proteolysis activities, explaining the slow transition between soluble and "classical" IB.

The data we report suggests that PdhS-mCherry is folded in aggregates resembling "non-classical" IB. The data supporting the folded state of PdhS in E. coli are that PdhS-mCherry (i) is soluble and forms multimers 
of homogeneous size, and (ii) is still able to interact with partners like the fumarase FumC and the response regulator DivK. The recent resolution of a complex between a histidine kinase and its cognate response regulator [19] strongly suggests that the dimerization and histidine-containing phosphotransfer (DHp) domain of the kinase needs to be folded to allow interaction with the response regulator. It is therefore predictable that at least the DHp domain of PdhS-mCherry is folded to allow interaction with DivK-YFP. Interestingly, we previously reported that $B$. abortus PdhS was able to colocalize with $B$. abortus fumarase FumC, but not with $C$. crescentus FumC [18], and here the recruitment of FumC proteins by PdhS-mCherry is consistent with this specificity (Fig. 6A and 6B). Moreover, it means that fusions to YFP are not all aspecifically associated to soluble aggregates of PdhS-mCherry resembling "nonclassical" IB.

A striking observation is the mobility of IbpA-YFP foci inside cells during the stationary phase $\left(\right.$ at $\left.t_{12}\right)$. This mobility is strongly decreased in late stationary cells $\left(t_{36}\right)$, where larger and brighter IbpA-YFP foci are observed at the bacterial poles. IbpA-YFP foci also move around in PdhS-mCherry aggregates producing cells at $\mathrm{t}_{12}$, until they meet PdhS-mCherry aggregates. The dynamic localization of IbpA-YFP suggests a model in which IbpA could scan the bacterial cell to bind to protein aggregates before taking part in a disaggregation process. This hypothesis is supported by the observation of a fading of PdhS-mCherry fluorescence when it colocalizes with IbpA-YFP, concomitantly with an increase of the diffuse mCherry fluorescent signal (Fig 5C, Additional File 1), suggesting that a fraction of PdhSmCherry is removed from the "non-classical" IB. It would be interesting to test whether IbpA-YFP dynamic intracellular distribution is dependent on cytoskeletal elements. It would also be interesting to colocalize the $\mathrm{IbpB}$ co-chaperone with IbpA, and to investigate the role of the IbpA fibrils [20] in the intracellular motion of IbpA. Indeed, IbpA fibril formation is inhibited by aggregated substrates [20], and here we observed that IbpA-YFP is moving until it reaches IB. The absence of systematic colocalization of IbpA-YFP with PdhSmCherry (Fig. 3B) suggests that IbpA does not tightly and systematically bind all types of protein aggregates in E. coli. Even when IbpA-YFP localizes to the same pole as PdhS-mCherry, the position of the two foci is clearly distinct (Fig. 4), compared to the overlap observed for PdhS-mCherry and DivK-YFP or B. abortus FumC-YFP (Fig. 6). This suggests that IbpA-YFP and PdhS-mCherry do not truly colocalize, like PdhS-mCherry with DivKYFP or FumC-YFP, which have been reported to directly bind to PdhS $[17,18]$.

\section{Conclusion}

PdhS-mCherry is a new example of a protein able to form soluble "non-classical" inclusion bodies in E. coli. Here we report a detailed characterization of these particular IB using several approaches. These IB are able to recruit partners of PdhS, suggesting that PdhS remains folded in these IB, at least during a first step of IB maturation. The "non-classical" IB are probably highly sensitive to proteolysis, since they are quickly cleared from the cells when the environmental conditions change. Time lapse analysis of $E$. coli cells containing PdhS-mCherry "non-classical" IB indicates that IbpAYFP foci move rapidly inside the bacteria until they reach fluorescent aggregates. The characterization of IbpA-YFP movement inside $E$. coli should be investigated further as it could indicate how the IbpA chaperone is able to scan the cytoplasm to recognize intracellular protein aggregates.

\section{Methods}

\section{Strains, plasmids and media}

E. coli strains MG1655 expressing the $i b p A$ coding sequence (CDS) fused to the enhanced version of YFP CDS (13) and S17-1, TOP10 and DH10B were grown in liquid Luria-Bertani (LB) broth medium at $37^{\circ} \mathrm{C}$. Antibiotics were used at the following concentrations when appropriate: kanamycin, $50 \mu \mathrm{g} / \mathrm{ml}$ and chloramphenicol, $20 \mu \mathrm{g} / \mathrm{ml}$. The $p d h S$ CDS was inserted in fusion with the $m$ Cherry CDS on a high-copy number plasmid, in the opposite orientation of the lac promoter, derived from the pBluescriptKS vector (Stratagene); this plasmid was named pCVDH07. The E. coli strains transformed with pCVDH07 were grown in liquid LB with kanamycin for times indicated in the text, without induction of gene expression for the PdhS-mCherry fusion. The growth was followed by measuring the optical density at $600 \mathrm{~nm}$.

\section{Microscopy}

For fluorescence imaging, E. coli S17-1 and MG1655 strains were placed on a microscope slide that was layered with $1 \%$ agarose containing either PBS or $1 \%$ agarose containing LB medium (40 g/l). Time-lapse microscopy was performed by placing strains on a microscope slide that was layered with a $1 \%$ agarose pad containing LB medium. Fluorescence corresponding to the mCherry reporter was observed at $583 \mathrm{~nm}$ using a TxRed filter. Fluorescence corresponding to the YFP signal was observed using an emission filter centered on 535 nanometers and an excitation from 490 to 510 nanometers. Samples were observed every 2 min using a Nikon i80 fluorescence microscope and the NIS software from Nikon with a Hamamatsu camera. 


\section{Protein extracts and Western blotting}

Cultures at the mid stationary phase (optical density at $600 \mathrm{~nm}$ of 1.5) were centrifuged and then washed twice in $20 \mathrm{mM}$ Tris- $\mathrm{HCl} 100 \mathrm{mM} \mathrm{NaCl}$ buffer at $\mathrm{pH}$ 7.9, lysed by sonication carried out over periods of $30 \mathrm{~s}$ with $1 \mathrm{~min}$ intervals in cooled tube on ice using a Branson sonifier 150. The cells were disrupted as observed microscopically to obtain total bacterial lysates that were centrifuged for 15 minutes at $13,000 \mathrm{rpm}$ at $4^{\circ} \mathrm{C}$. After centrifugation, the supernatant was harvested and considered as the soluble fraction of the bacterial cell lysate. The pellet was resuspended in PBS to reach the same volume as the supernatant, and was considered as the insoluble fraction. The soluble and insoluble fractions were then analysed by Western blot using polyclonal anti-DsRed antibodies (Clontech Laboratories, Inc) recognizing the mCherry protein, as previously reported (16).

\section{Gel filtration}

The soluble fraction of bacterial lysate $(500 \mu \mathrm{l})$ was injected into a HiPrep 16/60 Sephacryl S-500 HR column (GE Healthcare). The calibration curve was obtained using thyroglobulin $(669 \mathrm{kDa})$, apoferritin $(443$ $\mathrm{kDa})$ and amylase $(200 \mathrm{kDa})$. One milliliter fractions were collected and tested for the presence of the mCherry fluorochrome using a fluorimeter equipped with a TxRed filter. Positive fluorescent fractions were then tested by Western blot analysis using anti-DsRed antibodies.

\section{Additional material}

Additional file 1: Movement of IbpA-YFP in E. coli cells producing PdhS-mCherry. Time lapse movie of E. coli cells at stationary $\left(\mathrm{t}_{12}\right)$ phase, producing PdhS-mCherry (red) and IbpA-YFP (yellow). The time interval between two pictures is $2 \mathrm{~min}$.

Additional file 2: Time course of PdhS-mCherry production and gel permeation analysis of soluble extracts. PdhS-mCherry recombinant protein is detected by Western blot in the soluble fraction of E. coli expressing pdhS-mCherry fusion, and in the insoluble fraction in cells at late stationary phase (Figure S1). Western blot and fluorescence were used to detect PdhS-mCherry in gel permeation fractions, and allow the identification of a single peak corresponding to this fusion (Figure S2).

\begin{abstract}
Acknowledgements
We thank Ariel B. Lindner for kindly providing the E. coli strain expressing the chromosomal ibpA-yfp fusion and Etienne Maisonneuve for fruitful discussions. This work was supported by the FRFC (Collective Fundamental Research Fund, agreements 2.4521.04 and 2.4541.08) and by the University of Namur. C. Van der Henst and M. Deghelt held PhD fellowships from the FRIA (Industrial and Agricultural Research Training Fund). C. Charlier held a fellowship from the FRS-FNRS.
\end{abstract}

\section{Author details}

'Molecular Biology Research Unit (URBM), University of Namur (FUNDP), 61 rue de Bruxelles, 5000 Namur, Belgium. ${ }^{2}$ Theoretical and Structural Physical Chemistry Unit (UCPTS), University of Namur (FUNDP), 61 rue de Bruxelles, 5000 Namur, Belgium.

\section{Authors' contributions}

CVDH performed all experiments with the help of others, as indicated below, and drafted the manuscript. CC and JW performed to the gel permeation experiment. MD participated to the construction of the plasmid used for PdhS-mCherry production in E. coli. JYM contributed to the microscopy. J JL participated in the writing of the manuscript. XDB coordinated the study and finalized the manuscript. All authors read and approved the final manuscript.

Received: 14 July 2010 Accepted: 28 September 2010 Published: 28 September 2010

\section{References}

1. Speed MA, Wang DI, King J: Specific aggregation of partially folded polypeptide chains: the molecular basis of inclusion body composition. Nat Biotechnol 1996, 14(10):1283-1287.

2. Villaverde A, Carrio MM: Protein aggregation in recombinant bacteria: biological role of inclusion bodies. Biotechnol Lett 2003, 25(17):1385-1395.

3. Ventura S, Villaverde A: Protein quality in bacterial inclusion bodies. Trends Biotechnol 2006, 24(4):179-185.

4. Strandberg L, Enfors SO: Factors influencing inclusion body formation in the production of a fused protein in Escherichia coli. Appl Environ Microbiol 1991, 57(6):1669-1674.

5. Maisonneuve E, Ezraty B, Dukan S: Protein aggregates: an aging factor involved in cell death. J Bacteriol 2008, 190(18):6070-6075.

6. Kwiatkowska J, Matuszewska E, Kuczynska-Wisnik D, Laskowska E: Aggregation of Escherichia coli proteins during stationary phase depends on glucose and oxygen availability. Res Microbiol 2008, 159(910):651-657.

7. Carrio MM, Villaverde A: Construction and deconstruction of bacterial inclusion bodies. J Biotechnol 2002, 96(1):3-12.

8. Allen SP, Polazzi JO, Gierse JK, Easton AM: Two novel heat shock genes encoding proteins produced in response to heterologous protein expression in Escherichia coli. J Bacteriol 1992, 174(21):6938-6947.

9. Winkler J, Seybert A, Konig L, Pruggnaller S, Haselmann U, Sourjik V, Weiss M, Frangakis AS, Mogk A, Bukau B: Quantitative and spatio-temporal features of protein aggregation in Escherichia coli and consequences on protein quality control and cellular ageing. Embo J 29(5):910-923.

10. Kuczynska-Wisnik D, Kedzierska S, Matuszewska E, Lund P, Taylor A, Lipinska B, Laskowska E: The Escherichia coli small heat-shock proteins $\mathrm{IbpA}$ and $\mathrm{IbpB}$ prevent the aggregation of endogenous proteins denatured in vivo during extreme heat shock. Microbiology 2002, 148(Pt 6):1757-1765.

11. Lindner AB, Madden R, Demarez A, Stewart EJ, Taddei F: Asymmetric segregation of protein aggregates is associated with cellular aging and rejuvenation. Proc Natl Acad Sci USA 2008, 105(8):3076-3081.

12. Rokney A, Shagan M, Kessel M, Smith Y, Rosenshine I, Oppenheim AB: E. coli transports aggregated proteins to the poles by a specific and energy-dependent process. J Mol Biol 2009, 392(3):589-601.

13. Oberg K, Chrunyk BA, Wetzel R, Fink AL: Nativelike secondary structure in interleukin-1 beta inclusion bodies by attenuated total reflectance FTIR. Biochemistry 1994, 33(9):2628-2634.

14. Gonzalez-Montalban N, Garcia-Fruitos E, Ventura S, Aris A, Villaverde A: The chaperone DnaK controls the fractioning of functional protein between soluble and insoluble cell fractions in inclusion body-forming cells. Microb Cell Fact 2006, 5:26.

15. Stampolidis P, Kaderbhai NN, Kaderbhai MA: Periplasmically-exported lupanine hydroxylase undergoes transition from soluble to functiona inclusion bodies in Escherichia coli. Arch Biochem Biophys 2009, 484(1):8-15.

16. Jevsevar S, Gaberc-Porekar V, Fonda I, Podobnik B, Grdadolnik J, Menart V: Production of nonclassical inclusion bodies from which correctly folded protein can be extracted. Biotechnol Prog 2005, 21(2):632-639.

17. Hallez R, Mignolet J, Van Mullem V, Wery M, Vandenhaute J, Letesson JJ, Jacobs-Wagner $C$, De Bolle $X$ : The asymmetric distribution of the essential histidine kinase PdhS indicates a differentiation event in Brucella abortus. Embo J 2007, 26(5):1444-1455,

18. Mignolet J, Van der Henst C, Nicolas C, Deghelt M, Dotreppe D, Letesson JJ, De Bolle X: PdhS, an old-pole-localized histidine kinase, recruits the fumarase FumC in Brucella abortus. J Bacteriol 2010, 192(12):3235-3239. 
19. Casino $P$, Rubio V, Marina A: Structural insight into partner specificity and phosphoryl transfer in two-component signal transduction. Cell 2009, 139(2):325-336.

20. Ratajczak E, Strozecka J, Matuszewska M, Zietkiewicz S, Kuczynska-Wisnik D, Laskowska E, Liberek K: IbpA the small heat shock protein from Escherichia coli forms fibrils in the absence of its cochaperone IbpB. FEBS Lett 2010, 584(11):2253-2257.

doi:10.1186/1471-2180-10-248

Cite this article as: Van der Henst et al: Overproduced Brucella abortus PdhS-mCherry forms soluble aggregates in Escherichia coli, partially associating with mobile foci of IbpA-YFP. BMC Microbiology 2010 10:248.

Submit your next manuscript to BioMed Central and take full advantage of:

- Convenient online submission

- Thorough peer review

- No space constraints or color figure charges

- Immediate publication on acceptance

- Inclusion in PubMed, CAS, Scopus and Google Scholar

- Research which is freely available for redistribution

Submit your manuscript at www.biomedcentral.com/submit
Ciomed Central 